\title{
A Statistical Model to Estimate the Number of Registration Desks Required to Minimize Patient Wait Time in the Outpatient Department of a Multispecialty Hospital
}

\author{
Priya Darshini M Pedagandham ${ }^{1}$, Swapnil B Tak ${ }^{2}$
}

\begin{abstract}
Introduction: Gap between demand and limited availability of resources often lead to delays, which in turn affect the patient flow in a typical hospital setting. Registration of new patients at front desk is one such place. Delays in the registration process can have an unwanted effect on patient-hospital relationship. Hence, it is important to reduce the delay.

Aim: To identify various reasons for delay in the registration process of a multispecialty hospital, and develop a statistical model to estimate number of desks to be operated to reduce the wait time.

Materials and methods: In a period of two weeks, a total of 923 new patient registrations were observed to collect the time consumed for various events of the registration process. Correlation analysis and statistical modelling are used in the study.

Results and discussion: It was found that various factors such as literacy level of patients, mode of payments, age etc. do not show significant correlation with the registration time, except for the hourly patient load. The number of patients arriving in an hour (patient load) showed a significant correlation, suggesting that number of desks operated on regular basis was not sufficient to handle occasional high loads. A simple model that accounts for hourly patient load, estimating the number of desks to be operated in a given hour, to minimize the wait times at registration desk has been suggested.

Conclusion: The model indicates that in the current setting, most of the times the resources were either underutilized or overloaded. The delays in registration process can be reduced by operating optimal number of desks as determined by the proposed model (i.e. function of hourly patient load).

Keywords: Hospital, Patient load, Patient registration, Resource planning, Wait time.

International Journal of Research Foundation of Hospital and Healthcare Administration (2019): 10.5005/jp-journals-10035-1101
\end{abstract}

\section{INTRODUCTION}

A gap between the demand and availability of healthcare resources and maximization of their utility often leads to delays in the patient flow process. ${ }^{1}$ Typically, patients visiting a hospital to avail various services may have to wait at various stages, such as patient registration for consultation with doctor, diagnostics, admission into a ward, transfer between wards, and discharge. ${ }^{2}$ Depending upon the availability of required resources, the wait time can vary from few minutes to hours, which may result in displeasure among patients. ${ }^{3}$ Hence, it is important for the hospital administration to develop and implement better processes to reduce possible delays while optimizing the resource utilization.

In general, every patient has to go through registration process during their first visit to the hospital to avail services offered. ${ }^{4}$ Being the first step in the hospital-patient relation, delays in the registration process may affect the relationship. It is therefore important to monitor the registration process (besides various other processes resulting in delays) to minimize waiting time and to promote continuous improvement of the processes. Delays are typically due to a lack of synchronization between various activities. ${ }^{2}$ The present work aims to develop a solution to address delays in the new patient registration process of a multispecialty hospital.

Registration process is usually concerned with collection and recording of demographic details of patients along with their brief medical history. ${ }^{2}$ The process may vary a little from hospital to hospital. In the selected hospital, a patient when visiting for the first time is given a file with an empty registration form; the form
${ }^{1}$ Center for Health Management Studies and Research, Bharati Vidyapeeth Medical College, Bharati Vidyapeeth (Deemed to be University), Pune, Maharashtra, India

${ }^{2}$ Department of Preventive and Social Medicine, Bharati Vidyapeeth Medical College, Bharati Vidyapeeth (Deemed to be University), Pune, Maharashtra, India

Corresponding Author: Swapnil B Tak, Department of Preventive and Social Medicine, Bharati Vidyapeeth Medical College, Bharati Vidyapeeth (Deemed to be University), Pune, Maharashtra, India, Phone: +91 9822837373, e-mail: swapnil.tak@bharatividyapeeth.edu

How to cite this article: Pedagandham PDM, Tak SB. A Statistical Model to Estimate the Number of Registration Desks Required to Minimize Patient Wait Time in the Outpatient Department of a Multispecialty Hospital. Int J Res Foundation Hosp Healthc Adm 2019;7(1):24-28.

Source of support: Nil

Conflict of interest: None

has to be filled and submitted to registration desk staff. The form contains patient information such as demographics, drug allergies, and emergency contact details. The information collected is fed into the hospital information system (HIS) and stored permanently. A unique identification number (also referred to as medical record number (MRN)) is generated and tagged with the patient's details in the HIS. The MRN helps to store and retrieve patient's data from the HIS when required. Flowchart 1 depicts the work flow of new patient registration. It involves various steps such as inquiries about 
Flowchart 1: A schematic representation of the workflow of patient registration process in the selected hospital

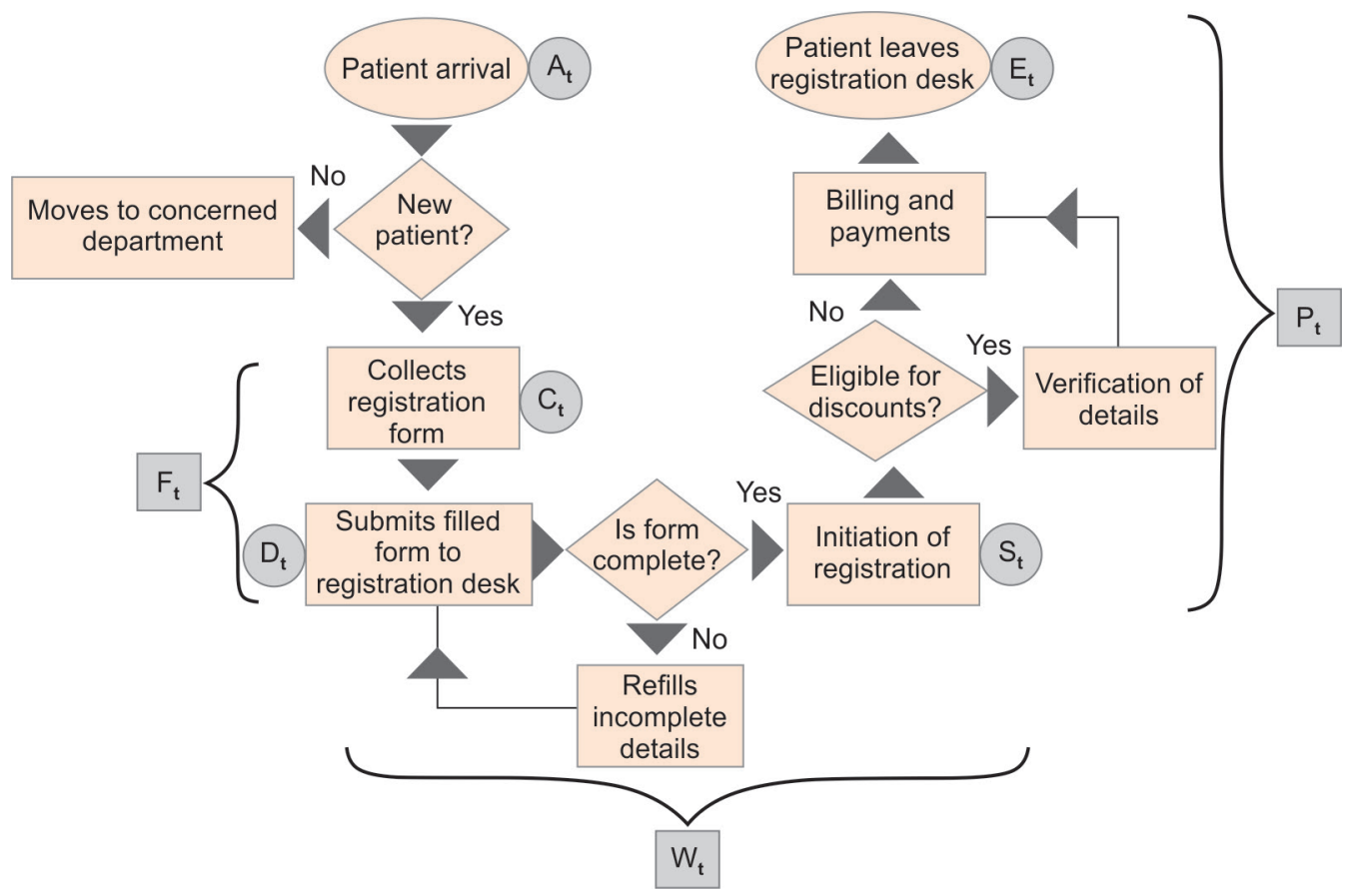

availability of doctors or services, collection and filling of various necessary forms, verification of the information by staff, generation of MRN, verification of patient's eligibility for discounts (if any), and payment of registration and/or consultation charges by cash or card transaction. Objective of the work is to measure the time taken for various subprocesses involved in the registration process, identify factors causing a delay in the registration process, and to develop a statistical model to improve the process (i.e., reduce wait times).

The rest of the article is organized as follows. Data collection methodology and analysis of data are discussed in Materials and Methods section. Results and Discussion section present the results of the study and findings are summarized in Conclusion section.

\section{Materials and Methods}

This is an observational study of registration process conducted in a multispeciality hospital for a period of two weeks between 9:00 and 17:00 hours. All the necessary permissions were taken from the hospital management for collection of the primary data. The time taken for the registration and its sub-processes was recorded. A digital clock with minutes as the least count was used to record the time of occurrence of various events. The time was recorded at the following stations as mentioned in Flowchart 1: arrival time of patient at the registration desk $\left(A_{t}\right)$, time at which the file was collected by the patient from the desk $\left(C_{t}\right)$, time at which the filled up form was submitted to the registration desk $\left(D_{\mathrm{t}}\right)$, time at which staff began digitization (i.e., entry of patient's data into HIS) $\left(S_{t}\right)$, and time at which the registration process was completed $\left(E_{\mathrm{t}}\right)$. In addition, various attributes pertaining to patients such as age, literacy level (qualification), mode of payment (cash or card), and eligibility for discounts were also recorded for further analysis.
The files are processed at the registration counters in firstin-first-out manner. Depending upon the patient load, the forms submitted to the registration desk may take some time to be fed into HIS, during which patients have to wait at the desk. Time taken by a patient to fill various forms (denoted as form time, $F_{t}$, Flowchart 1), time elapsed between submission of forms and initiation of digitization (wait time, $W_{\mathrm{t}}$ ) and the time taken by the staff to digitize the information and to book appointment (process time, $P_{\mathrm{t}}$ ) were computed from the recorded information as

$$
\begin{aligned}
& F_{\mathrm{t}}=D_{\mathrm{t}}-C_{\mathrm{t}} \\
& W_{\mathrm{t}}=S_{\mathrm{t}}-D_{\mathrm{t}} \\
& P_{\mathrm{t}}=E_{\mathrm{t}}-S_{\mathrm{t}}
\end{aligned}
$$

The total registration time $\left(R_{\mathrm{t}}\right)$ is

$$
R_{\mathrm{t}}=F_{\mathrm{t}}+W_{\mathrm{t}}+P_{\mathrm{t}}
$$

\section{Statistical Methodology}

Correlation is a statistical measure that defines the association between a pair of variables. ${ }^{5,6}$ Pearson correlation coeffcient " $r$ " assumes values $|r|=0$ for a pair of variables with no correlation and 1 for highly interdependent variables. Sign of the $r$ indicates either a positive or negative relation between the variables (e.g., -1 indicates strong anti-correlation). On the basis of the value of $|r|$, the correlation between a pair of variables is classified into following categories. Very high correlation: $0.9<|r|<1.0$; high correlation: $0.7<|r|<0.89$; moderate correlation: $0.5<|r|<0.69$; low correlation: $0.3<|r|<0.49$; little (if any) correlation: $0.0<|r|<0.29$. 
Scatter plot is a graphical representation of relation between a pair of variables (e.g., $x$ and $y$ ). If $x$ and $y$ are strongly correlated, then the $x$ vs $y$ data fall along a line; otherwise it scatters all over. The scatter plot is a useful tool to identify the cause and effect relation. ${ }^{5,6}$

Box plot is a graphical presentation of characteristics of data such as median, first and third quartiles $\left(Q_{1}\right.$ and $\left.Q_{3}\right)$, inter quartile range (IQR $=Q_{3}-Q_{1}$ ), and outliers. It also indicates variability and skewness in the data. It enables one to examine and compare two or more sets of data graphically. ${ }^{5}$

\section{Results and Discussion}

Data pertaining to 923 new patient registrations were collected during two weeks period, and computed the $F_{\mathrm{t}}, W_{\mathrm{t}}, P_{\mathrm{t}}$, and $R_{\mathrm{t}}$ for each registration. The data were pre-processed to remove the outliers using box and whisker method. ${ }^{5}$ Data points below $\left(Q_{1}-1.5 \mathrm{IQR}\right)$ or above $\left(Q_{3}+1.5 \mathrm{IQR}\right)$ were marked as outliers. The identified outliers were replaced with the corresponding data of previous registration.

Figure 1 shows box-plots of $F_{\mathrm{t}}, W_{\mathrm{t}}, P_{\mathrm{t}}$, and $R_{\mathrm{t}}$ for all the registrations. Variability of $P_{\mathrm{t}}$ is the minimum, followed by $F_{\mathrm{t}}, W_{\mathrm{t}}$, and $R_{\mathrm{t}}$. Minimum values of the $F_{\mathrm{t}}, W_{\mathrm{t}}, P_{\mathrm{t}}$, and $R_{\mathrm{t}}$ were $1,2,3$, and 4 minutes, respectively. Mean values of the respective $F_{\mathrm{t}^{\prime}}, W_{\mathrm{t}^{\prime}} P_{\mathrm{t}^{\prime}}$ and $R_{\mathrm{t}}$ are found to be 9.5, 5, 7.5, and 20 minutes.

A correlation analysis of the time consumed for the registration and the various factors associated with the process helps identify important factors affecting the registration process. It enables to improve the process by working on variables that have a dominant effect (i.e., high values of $r$ ).

The correlation was computed between hourly mean process time and various attributes such as hourly number of card transactions, number of registrations availing discounts, number of geriatric patients, and process interruptions. The respective correlation coefficients $|r|$ were $-0.04,0.21,0.23$, and 0.29 . A weak correlation shown by these attributes signifies that none of these attributes affect the process time. This is in agreement with the minimum variability shown by the $P_{\mathrm{t}}$ in Figure 1 .

Figure 2 shows patient load as a function of the clock time from 9:00 to 16:00 hours. The patient load (i.e., the number of patients who undergo registration) peaks every day at 11:00 and 15:00 hours.

As shown in Figure 3, the hourly mean $R_{\mathrm{t}}$ also peaks around the same time as the patient load, suggesting an association between the patient load and the $R_{\mathrm{t}}$.

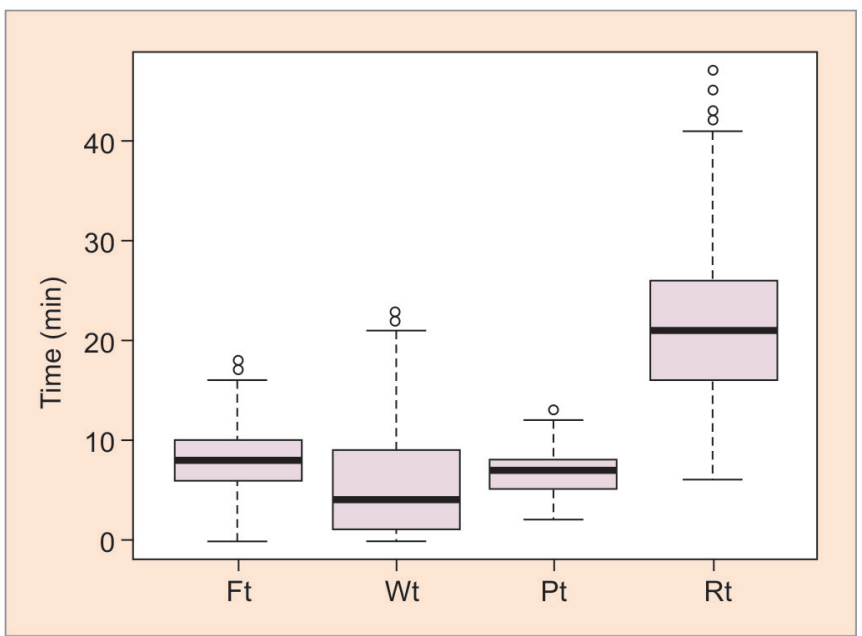

Fig. 1: A box plot showing the distribution of various times: $F_{\mathrm{t}^{\prime}} W_{\mathrm{t}}, P_{\mathrm{t}}$, and $R_{\mathrm{t}}$ based on the data of 923 patients

\section{Relation between Patient Load and Registration Time}

To study the effect of patient load on registration time, the correlation between the hourly patient load and the hourly mean $R_{\mathrm{t}}$ was calculated. A moderate correlation was found, with an $r$ value of 0.54 , as depicted by the scatter plot in Figure 4.

Furthermore, correlations between the hourly patient load and the time taken for various sub-processes were also computed. The $r$ value obtained for the $F_{\mathrm{t}}, W_{\mathrm{t}}$, and $P_{\mathrm{t}} \mathrm{vs}$ the number of patients were 0.34 (weak correlation), 0.57 (moderate correlation), and 0.05 (no correlation), respectively. It implies that the wait time (i.e., the delay in registration process) is associated with the patient load, and also suggests that the number of registration counters operated (in general, three counters) may not be sufficient for catering to the incoming patients. A simple solution (but not the optimal one) would be to operate more number of registration counters than the typical number of desks operated, but it may lead to underutilization of the resources during the off-peak hours.

\section{Optimal Number of Registration Counters}

In the selected hospital, typically, three registration desks were operated on a regular basis, which was resulting in either delays in the registration process or underutilization of the resources depending upon the patient load. It was therefore required to estimate the optimum number of registration desks to be operated as a function of patient load to improve the resource utilization and to reduce delays. A simple calculation procedure was proposed to estimate the optimal number of counters to be operated as a function of the hourly patient load.

The number of counters to be operated is defined as

$$
\text { Number of counters = Hourly patient load / }
$$$$
\text { Hourly number of registrations per desk }
$$

It is to be noted that mean and standard deviation of the process time are 7.06 and 2.08 minutes, respectively. If only the mean process time is considered, the hourly number of registrations per desk will be

\section{Hourly number of registrations per desk \\ $=60$ minutes $/$ mean process time in minutes.}

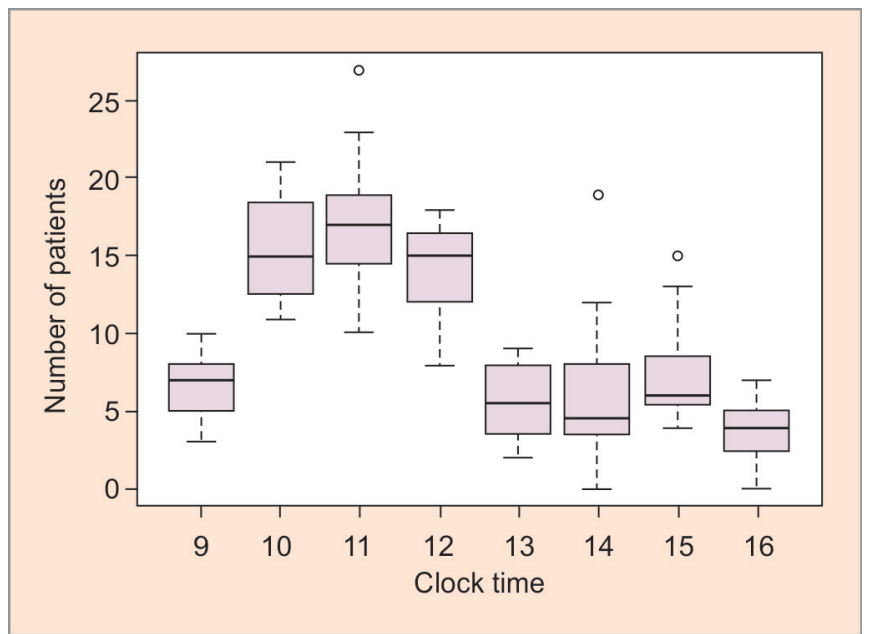

Fig. 2: A box plot showing the hourly number of patients (NOP) registered. Patient load and variability were high during $10-12$ and $14-15$ hours 


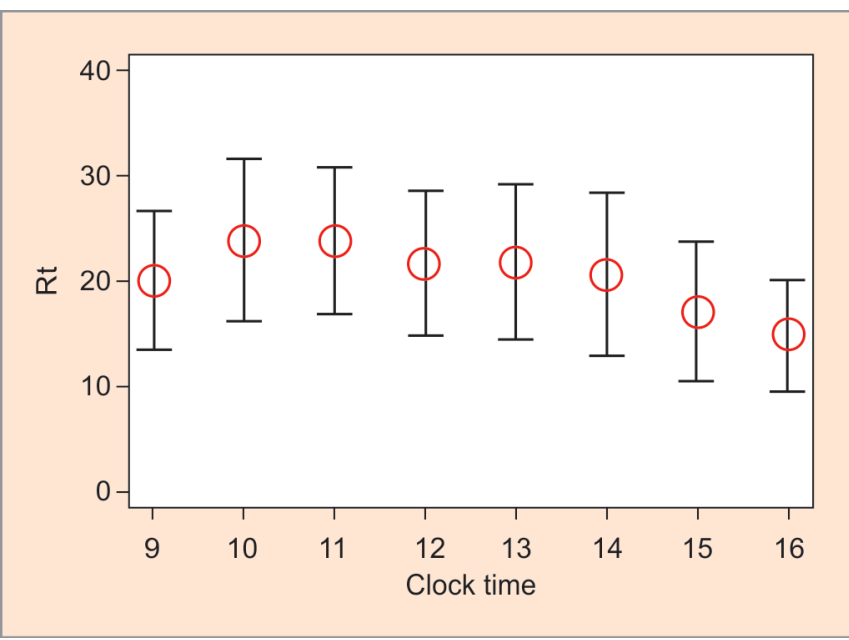

Fig. 3: Hourly mean registration time as function of clock time

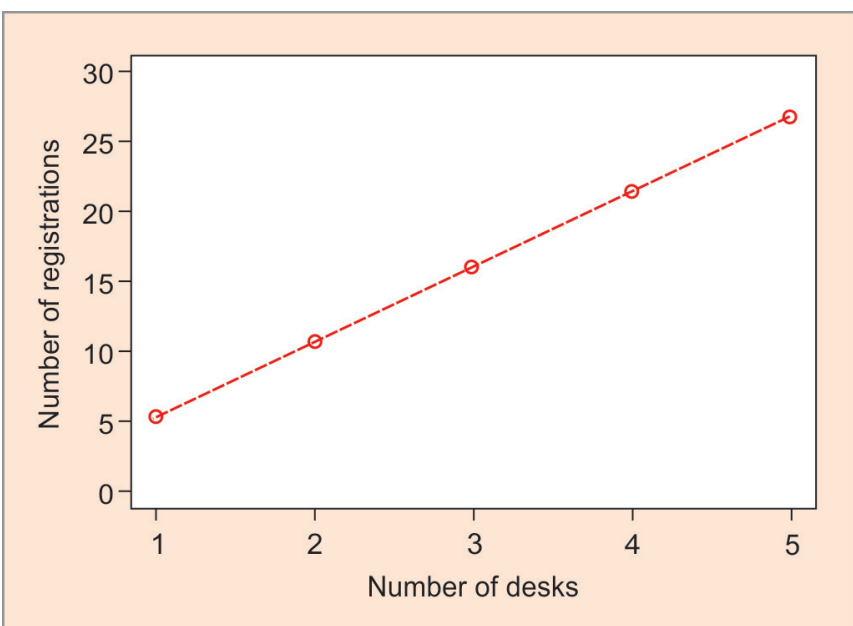

Fig. 5: The number of registration desks to be operated as a function of the patient load. Circles represent the model predictions that account for variability along with the mean process time (eqn 6)

The variability in the process indicated by a finite value of standard deviation needs to be accounted. The hourly number of registrations per desk that accounts for $95 \%$ of variability in the process is given by

\section{Hourly number of registrations per desk}

$=60 /$ (mean process time $+2 \times$ standard deviation $)$

Solid line in Figure 5 shows the number of counters to be operated as per eqn 5 and 6 .

In Figure 6, the optimum number of counters to be operated as a function of patient load (eqn 6) in a given hour is shown. For instance, the optimal number of desks, indicated by circles (Monday) and triangles (Tuesday), do not agree with the dotted line (3 number of desks) most of the times. The proposed model suggests operating four desks at 10-11 hours on Monday, but only one desk for 13-16 hours on Tuesday to optimize the resource utilization. To verify the model estimates for the optimal number of desks, hourly mean wait times observed on the same days are plotted in Figure 7. On Monday, wait times are high for 10 and 11 hours, for which the model suggests to operate 4 desks. Hence the proposed model is

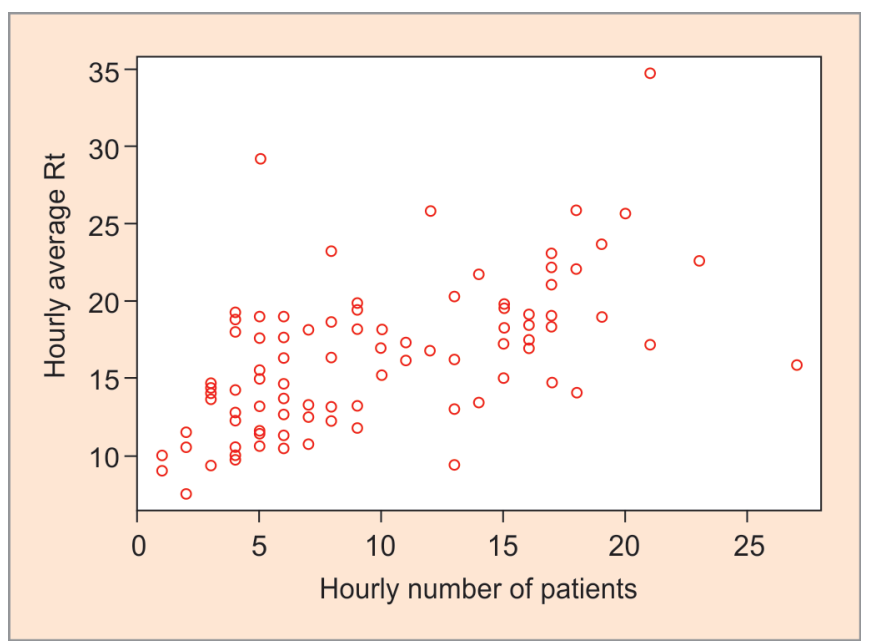

Fig. 4: A scatter plot showing the relation between hourly average $R_{\mathrm{t}}$ and patient load at the corresponding time

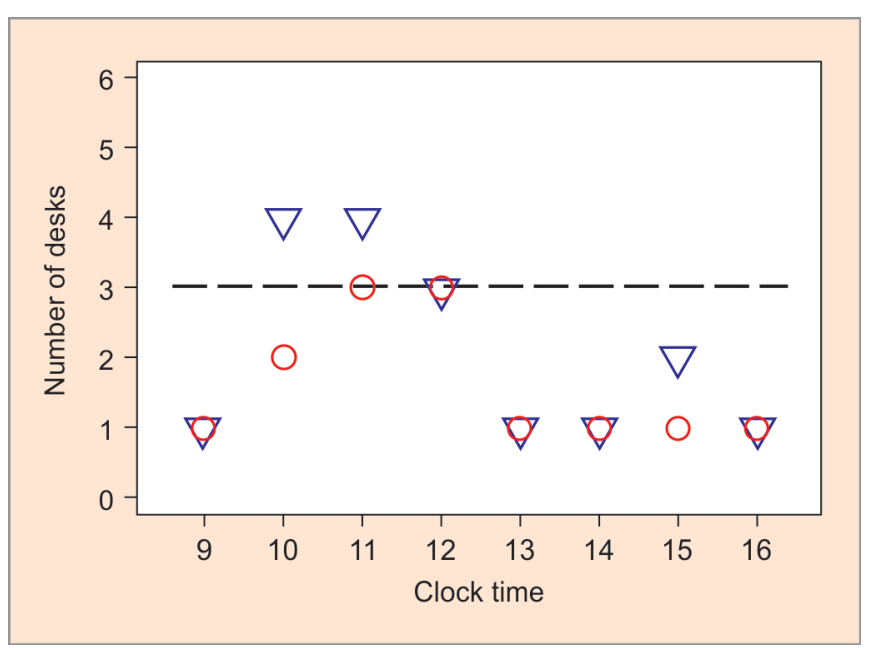

Fig. 6: A comparison of the model suggested the number of desks to be operated (circles and triangles) with the number of desks operated on a regular basis (dashed line) as a function of the clock time. The discrepancy between the two indicates suboptimal utilization of the resources

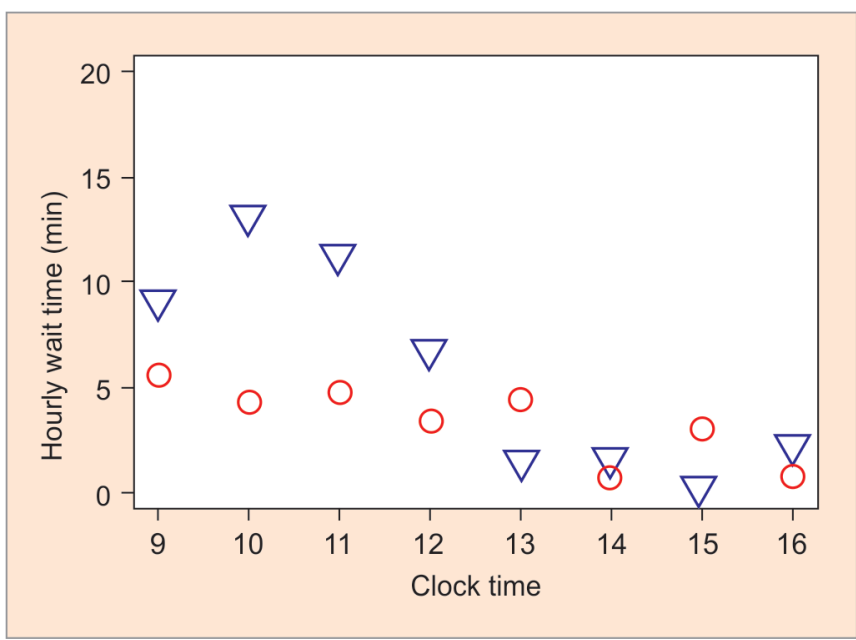

Fig. 7: The wait time in minutes as function of the clock time for two days: Monday (triangles) and Tuesday (circles) for which the model predictions are presented in Figure 6 
valid, and can be utilized to allocate the resources for registration desk such that the delays in the registration process can be reduced, while maintaining optimal utilization of resources.

\section{CONCLUSION}

Delay in the registration process is one of the factors that can affect the patient flow in a hospital. In this study, data such as patient demographics and time consumed for registration process were collected for the new patient registrations in a time period of two weeks. Statistical analysis of the data suggested that factors such as patient age, qualification, literacy level, and mode of payment do not contribute to the delays in the registration process. The hourly patient load and registration time (wait time) were moderately correlated, indicating that non-operation of a required number of registration desks is the reason for delays in registration process. In this work, a simple model estimating the number of desks to be operated to minimize the delays in the registration process was proposed. The proposed model indicates that the registration desk resources were underutilized most of the time, and were not sufficient to handle patient loads during peak hours. The model predictions are supported by the observed wait times. The delays in registration process can be reduced by operating optimal number of desks, as determined by the model as a function of the hourly patient load.

\section{ACKNOWLedgments}

Authors are grateful to Dr Mahesh Mynam for his contribution and support in this work. They are also thankful to Dr HS Acharya for his valuable inputs.

\section{References}

1. Su $Q, Y a o X$, et al. Hospital registration process reengineering using simulation method. J Healthc Eng 2010;1(1):67-82.

2. Hall $R$, Belson $D$, et al. Modeling patient flows through the health care system. Patient Flow. Boston, MA: Springer; 2013; pp. 3-42.

3. Jones $P$, Peppiatt $E$. Managing perceptions of waiting times in service queues. Int J Serv Ind Manag 1996 Dec 1;7(5):47-61. DOI: 10.1108/09564239610149957.

4. Mohd A, Chakravarty A. Patient satisfaction with services of the outpatient department. Med J Armed Forces India 2014 Jul 1;70(3):237-242. DOI: 10.1016/j.mjafi.2013.06.010.

5. Chernick MR, Friis RH. Introductory Biostatistics for the Health Sciences: Modern Applications Including Bootstrap. John Wiley and Sons; 2003 Jun 24

6. Van Belle G, Fisher LD, et al. Biostatistics: A Methodology for the Health Sciences. John Wiley and Sons; 2004 Oct 20.

7. Asuero AG, Sayago A, et al. The correlation coefficient: An overview. Crit Rev Anal Chem 2006 Jan 1;36(1):41-59. DOI: 10.1080/10408340500526766. 